Bull. Korean Math. Soc. 49 (2012), No. 4, pp. 669-684

http://dx.doi.org/10.4134/BKMS.2012.49.4.669

\title{
DEGENERATE SEMILINEAR ELLIPTIC PROBLEMS NEAR RESONANCE WITH A NONPRINCIPAL EIGENVALUE
}

\author{
Hong-Min Suo and Chun-Lei Tang
}

\begin{abstract}
Using the minimax methods in critical point theory, we study the multiplicity of solutions for a class of degenerate Dirichlet problem in the case near resonance.
\end{abstract}

\section{Introduction and main results}

Consider degenerate semilinear elliptic equation of the form

$$
\begin{cases}-\operatorname{div}(a(x) \nabla u)=\lambda u+f(x, u)+h(x) & \text { in } \Omega, \\ u=0, & \text { on } \partial \Omega,\end{cases}
$$

where $\Omega$ is a bounded domain in $\mathbb{R}^{N}(N \geq 2)$ with smooth boundary $\partial \Omega$, $a$ is a nonnegative measurable weight on $\Omega, \lambda \in \mathbb{R}, h \in L^{2}(\Omega)$, and $f: \Omega \times \mathbb{R} \rightarrow \mathbb{R}$ is a Carathéodory function and satisfies the following assumption.

(A) There exist constants $C>0$ and $q \in(1,2)$ such that

$$
|f(x, t)| \leq C\left(1+|t|^{q-1}\right) .
$$

Problem (1) was introduced as models for several physical phenomena related to equilibrium of continuous media which somewhere are perfect insulators or perfect conductors (see [5]).

Assume that

$\left(\mathrm{H}_{\alpha}\right) \quad a \in L_{l o c}^{1}(\Omega)$, and there exists a constant $\alpha \in[0,+\infty)$ such that

$$
\lim \inf _{x \rightarrow z}|x-z|^{-\alpha} a(x)>0
$$

for every $z \in \bar{\Omega}$.

From this assumption, Caldiroli and Musina in [2] have proved that there exist

Received March 2, 2011.

2010 Mathematics Subject Classification. 35J20, 35J25, 35J61, 35J70.

Key words and phrases. degenerate elliptic equations, near resonance, Dirichlet problem, eigenvalue, saddle point.

Supported by the National Natural Science Foundation of China (No. 11071198), by National Commission of Ethnic Affairs Science Foundation of China (No.[2011]02), by Natural Science Foundation of the Education Department of GuiZhou Province (No.[2010]305). 
a finite set $Z=\left\{z_{1}, z_{2}, \ldots, z_{k}\right\} \subset \bar{\Omega}$ and numbers $\gamma, \delta>0$ such that the balls $B_{i}=B_{\gamma}\left(z_{i}\right)(i=1,2, \ldots, k)$ are mutually disjoint and

$$
a(x) \geq \delta\left|x-z_{i}\right|^{\alpha}, \quad \forall x \in B_{i}, i=1,2, \ldots, k,
$$

and

$$
a(x) \geq \delta, \quad \forall x \in \bar{\Omega} \backslash \bigcup_{i=1}^{k} B_{i} .
$$

This says that the elliptic operator in problem (1) is degenerate and the set $Z_{a}=\{x \in \bar{\Omega}: a(x)=0\}$ is finite.

By the presence of function $a$, weak solutions of equation (1) must be found in a suitable space. To this purpose, we define the space $H_{0}^{1}(\Omega, a)$ (see [2]) as the closures of $C_{0}^{\infty}(\Omega)$ with the norm

$$
\|u\|=\left(\int_{\Omega} a(x)|\nabla u|^{2} d x\right)^{\frac{1}{2}}
$$

for $u \in C_{0}^{\infty}(\Omega)$. In fact, $H_{0}^{1}(\Omega, a)$ is a Hilbert space with the inner product

$$
\langle u, v\rangle=\int_{\Omega} a(x)(\nabla u, \nabla v) d x
$$

for $u, v \in H_{0}^{1}(\Omega, a)$. Moreover, we have the following lemma.

Lemma 1 (Proposition 3.2, [2]). Assume that $\left(\mathrm{H}_{\alpha}\right)$ holds for some $\alpha \in(0,2]$. Then $H_{0}^{1}(\Omega, a) \hookrightarrow L^{p}(\Omega)$ is compact if $p \in\left[1,2_{\alpha}^{*}\right)$, where $2_{\alpha}^{*}=\frac{2 N}{N-2+\alpha}$.

From this lemma, it is not difficult to check that the associated functional of problem (1) $J: H_{0}^{1}(\Omega, a) \rightarrow \mathbb{R}$ defined as follows

$$
J(u)=\frac{1}{2} \int_{\Omega}\left(a(x)|\nabla u|^{2}-\lambda u^{2}\right) d x-\int_{\Omega} F(x, u) d x-\int_{\Omega} h u d x,
$$

is of class $C^{1}$, where $F(x, t)=\int_{0}^{t} f(x, s) d s$. And

$$
\left.\left\langle J^{\prime}(u), v\right)\right\rangle=\int_{\Omega} a(x)(\nabla u, \nabla v) d x-\lambda \int_{\Omega} u v d x-\int_{\Omega} f(x, u) v d x-\int_{\Omega} h v d x
$$

for $u, v \in H_{0}^{1}(\Omega, a)$. Furthermore, the weak solutions of system (1) are exactly the critical points of $J$ in $H_{0}^{1}(\Omega, a)$.

In addition, from Lemma 1 it follows that the operator $L$ defined by $L u:=$ $-\operatorname{div}(a(x) \nabla u)$ fits into the standard spectral theory for compact self-adjoint operators. Then there exists an increasing unbounded sequence of positive eigenvalues $0<\lambda_{1}(a)<\lambda_{2}(a)<\cdots<\lambda_{k}(a)<\cdots$ (see [2]). Denote by $E_{k}=\operatorname{ker}\left(L-\lambda_{k}(a)\right)$ the eigenspace corresponding to eigenvalue $\lambda_{k}(a)\left(k \in \mathbb{N}^{+}\right)$, then $E_{k}$ is a finite dimension space and we denote by $H_{k}=E_{1} \oplus E_{2} \oplus \cdots \oplus E_{k}$.

There are many results on multiplicity of solutions for non-degenerate equations like problem (1) approaching the first eigenvalue of corresponding linear problem. For instance, $[13,14,1,9,4,3]$ considered this problem in one or 
higher dimension via bifurcation theory or degree theory. As for results via variational methods, the readers are referred to $[11,10,16,6]$.

Results for higher eigenvalues were obtained in [9], [13] and [7]. Where [9] only considered the one-dimensional case via bifurcation from infinity and degree theory. [13] used bifurcation theory to deal with the eigenvalues of odd multiplicity. Recently, in [7], de Paiva and Massa considered this problem in any spatial dimension, and proved that there exist at least two solutions near resonance with any nonprincipal eigenvalue.

In the present paper, we extend the main results of [7] to the variational degenerate elliptic problem (1) by Local Saddle Point Theorem [12, 8] and Mountain Pass Lemma. Our main results are the following theorems.

Theorem 1. Suppose that $\left(\mathrm{H}_{\alpha}\right)$ holds for some $\alpha \in(0,2)$. Assume that $f$ satisfies (A) and the following condition

$$
\lim _{|t| \rightarrow \infty} \frac{f(x, t) t}{|t|}=+\infty
$$

uniformly for $x \in \Omega$. Then there exists $\delta_{0}>0$ such that for every $\lambda \in\left(\lambda_{k}(a)-\right.$ $\left.\delta_{0}, \lambda_{k}(a)\right)$, where $k \geq 2$, the problem (1) has at least two solutions.

Theorem 2. Suppose that $\left(\mathrm{H}_{\alpha}\right)$ holds for some $\alpha \in(0,2), f$ satisfies $(\mathrm{A})$ and the following condition

$$
\lim _{|t| \rightarrow \infty} F(x, t)=+\infty
$$

uniformly for $x \in \Omega$. Assume that

$$
\int_{\Omega} h \phi d x=0, \quad \forall \phi \in E_{k} .
$$

Then there exists $\delta_{1}>0$ such that for every $\lambda \in\left(\lambda_{k}(a)-\delta_{1}, \lambda_{k}(a)\right)$, where $k \geq 2$, the problem (1) has at least two solutions.

Theorem 3. Suppose that $\left(\mathrm{H}_{\alpha}\right)$ holds for some $\alpha \in(0,2)$. Assume that $f$ satisfies (A) and the following condition

$$
\lim _{|t| \rightarrow \infty} \frac{f(x, t) t}{|t|}=-\infty
$$

uniformly for $x \in \Omega$. Then there exists $\delta_{2}>0$ such that for every $\lambda \in$ $\left(\lambda_{k}(a), \lambda_{k}(a)+\delta_{2}\right)$, where $k \geq 2$, the problem (1) has at least two solutions.

Theorem 4. Suppose that $\left(\mathrm{H}_{\alpha}\right)$ holds for some $\alpha \in(0,2), f$ satisfies (A) and the following condition

$$
\lim _{|t| \rightarrow \infty} F(x, t)=-\infty
$$

uniformly for $x \in \Omega$. Assume that

$$
\int_{\Omega} h \phi d x=0, \quad \forall \phi \in E_{k} .
$$


Then there exists $\delta_{3}>0$ such that for every $\lambda \in\left(\lambda_{k}(a), \lambda_{k}(a)+\delta_{3}\right)$, where $k \geq 2$, the problem (1) has at least two solutions.

In order to prove our results, we need two abstract results as follows.

Theorem A (Link Theorem [15]). Let $H$ be a Hilbert space. Suppose that $J \in C^{1}(H, \mathbb{R})$ satisfies the $(P S)$ condition. Consider a closed subset $S \subset H$ and a submanifold $Q \subset H$ with relative boundary $\partial Q$. Suppose that

(i) $S$ and $\partial Q$ link,

(ii) $\alpha=\inf _{u \in S} J(u)>\sup _{u \in \partial Q} J(u)=\alpha_{0}$.

Let

then the number

$$
\Gamma=\left\{h \in C^{0}(H, H):\left.h\right|_{\partial Q}=i d\right\}
$$

$$
\beta=\inf _{h \in \Gamma} \sup _{u \in Q} J(h(u))
$$

defines a critical value $\beta \geq \alpha$ of $J$.

Theorem B (Local Saddle Point Theorem $[12,8]$ ). Let $H=X_{1} \oplus X_{2}$ be a Hilbert space where $X_{1}$ has finite dimension, $J \in C^{1}(H, \mathbb{R})$ satisfying the $(P S)$ condition and such that for given $\rho_{1}, \rho_{2}>0$,

$$
\sup _{u \in \rho_{1} S_{1}} J(u)<a=\inf _{u \in \rho_{2} B_{2}} J(u) \leq b=\sup _{u \in \rho_{1} B_{1}} J(u)<\inf _{u \in \rho_{2} S_{2}} J(u),
$$

where $B_{i}$ and $S_{i}$ represent the unit ball and the unit sphere in $X_{i}, i=1,2$. Then there exists a critical point $u_{0}$ such that $J\left(u_{0}\right) \in[a, b]$.

\section{Proof of theorems}

Define

$$
\begin{gathered}
B_{k-1}=\left\{u \in H_{k-1}:\|u\| \leq 1\right\}, \quad B_{k}=\left\{u \in H_{k}:\|u\| \leq 1\right\}, \\
B_{k}^{\perp}=\left\{u \in H_{k}^{\perp}:\|u\| \leq 1\right\},
\end{gathered}
$$

and $S_{k-1}, S_{k}, S_{k}^{\perp}$ are respectively their relative boundaries.

By assumption (A) and Lemma 1, one has

$$
\left|\int_{\Omega} F(x, u) d x\right| \leq C\left(1+\|u\|^{q}\right) .
$$

Moreover, denote by $\lambda_{k}=\lambda_{k}(a)$ for short, we have

$$
\begin{gathered}
\int_{\Omega} u^{2} d x \geq \frac{1}{\lambda_{k}}\|u\|^{2} \text { for } u \in H_{k}, \\
\int_{\Omega} u^{2} d x \leq \frac{1}{\lambda_{k+1}}\|u\|^{2} \text { for } u \in H_{k}^{\perp}, \\
\left|\int_{\Omega} h u d x\right| \leq\|h\|_{L^{2}}\|u\|_{L^{2}} \leq S\|h\|_{L^{2}}\|u\|,
\end{gathered}
$$

where $S$ is the best embedding constant. 
Proof of Theorem 1. The proof will be divided into four steps.

Step 1. For $\lambda \in\left(\lambda_{k-1}, \lambda_{k}\right)$, the functional $J$ satisfies the $(P S)$ condition.

Using (9) and (10) we have

$$
\begin{gathered}
\int_{\Omega} a(x)|\nabla u|^{2} d x-\lambda \int_{\Omega}|u|^{2} d x \leq \frac{\lambda_{k-1}-\lambda}{\lambda_{k-1}}\|u\|^{2}, \quad \forall u \in H_{k-1}, \\
\int_{\Omega} a(x)|\nabla u|^{2} d x-\lambda \int_{\Omega}|u|^{2} d x \geq \frac{\lambda_{k}-\lambda}{\lambda_{k}}\|u\|^{2}, \quad \forall u \in H_{k-1}^{\perp} .
\end{gathered}
$$

Let $\left\{u_{n}\right\} \subset H_{0}^{1}(\Omega, a)$ such that $\left\{J\left(u_{n}\right)\right\}$ is bounded and $J^{\prime}\left(u_{n}\right) \rightarrow 0$ as $n \rightarrow \infty$. We first prove that $\left\{u_{n}\right\}$ is bounded. By negation, suppose that $\left\|u_{n}\right\| \rightarrow \infty$ as $n \rightarrow \infty$. Let $u_{n}=v_{n}+w_{n} \in H_{k-1} \oplus H_{k-1}^{\perp}$. From Hölder inequality and (11), we have

$$
\begin{aligned}
& \left\langle J^{\prime}\left(u_{n}\right),\left(-v_{n}\right)\right\rangle \\
= & \lambda \int_{\Omega} u_{n} v_{n} d x-\int_{\Omega} a(x) \nabla u_{n} \cdot \nabla v_{n} d x+\int_{\Omega} f\left(x, u_{n}\right) v_{n} d x+\int_{\Omega} h v_{n} d x \\
\geq & \frac{\lambda-\lambda_{k-1}}{\lambda_{k-1}}\left\|v_{n}\right\|^{2}-C \int_{\Omega}\left(1+\left|u_{n}\right|^{q-1}\right)\left|v_{n}\right| d x-S\|h\|_{L^{2}}\left\|v_{n}\right\| \\
\geq & \frac{\lambda-\lambda_{k-1}}{\lambda_{k-1}}\left\|v_{n}\right\|^{2}-C\left\|v_{n}\right\|_{L^{1}}-C\left\|u_{n}\right\|_{L^{q}}^{q-1}\left\|v_{n}\right\|_{L^{q}}-S\|h\|_{L^{2}}\left\|v_{n}\right\| \\
\geq & \frac{\lambda-\lambda_{k-1}}{\lambda_{k-1}}\left\|v_{n}\right\|^{2}-C S\left\|v_{n}\right\|-C S^{q}\left\|u_{n}\right\|^{q-1}\left\|v_{n}\right\|-S\|h\|_{L^{2}}\left\|v_{n}\right\|,
\end{aligned}
$$

dividing the above inequality by $\left\|u_{n}\right\|^{2}$, noting that $\frac{\left\langle J^{\prime}\left(u_{n}\right),-v_{n}\right\rangle}{\left\|v_{n}\right\|} \rightarrow 0$, we have

$$
\frac{\left\|v_{n}\right\|}{\left\|u_{n}\right\|} \rightarrow 0
$$

as $n \rightarrow \infty$. Similarly, one has

$$
\frac{\left\|w_{n}\right\|}{\left\|u_{n}\right\|} \rightarrow 0
$$

as $n \rightarrow \infty$. Hence,

$$
1=\frac{\left\|u_{n}\right\|}{\left\|u_{n}\right\|} \leq \frac{\left\|w_{n}\right\|+\left\|v_{n}\right\|}{\left\|u_{n}\right\|} \rightarrow 0 \quad \text { as } n \rightarrow \infty,
$$

which is a contradiction. So $\left\{u_{n}\right\}$ is bounded.

In the following, we will prove that $\left\{u_{n}\right\}$ has a convergent subsequence. Let

$$
I(u)=\int_{\Omega} F(x, u) d x, \forall u \in H_{0}^{1}(\Omega, a) .
$$

Obviously, $I \in C^{1}\left(H_{0}^{1}(\Omega, a), \mathbb{R}\right)$ and

$$
\left\langle I^{\prime}(u), v\right\rangle=\int_{\Omega} f(x, u) v d x, \forall u, v \in H_{0}^{1}(\Omega, a) .
$$


Moreover, $I^{\prime}: H_{0}^{1}(\Omega, a) \rightarrow\left(H_{0}^{1}(\Omega, a)\right)^{*}$ is a compact operator by Lemma 1 . Since $\left\{u_{n}\right\}$ is bounded, i.e., there exists $M>0$ such that $\left\|u_{n}\right\| \leq M$. Then there exist a subsequence $\left\{u_{n_{k}}\right\} \subset\left\{u_{n}\right\}$ and $u \in H_{0}^{1}(\Omega, a)$ such that $I^{\prime}\left(u_{n_{k}}\right) \rightarrow$ $I^{\prime}(u)$. Denote by $u_{n_{k}}=\psi_{n_{k}}+\phi_{n_{k}} \in H_{k-1} \oplus H_{k-1}^{\perp}$, by (12) one has

$$
\begin{aligned}
& \left\langle J^{\prime}\left(u_{n_{k}}\right)-J^{\prime}\left(u_{n_{j}}\right), \psi_{n_{j}}-\psi_{n_{k}}\right\rangle \\
= & -\int_{\Omega} a(x)\left|\nabla\left(\psi_{n_{k}}-\psi_{n_{j}}\right)\right|^{2} d x+\lambda \int_{\Omega}\left(\psi_{n_{k}}-\psi_{n_{j}}\right)^{2} d x \\
& -\left\langle I^{\prime}\left(u_{n_{k}}\right)-I^{\prime}\left(u_{n_{j}}\right), \psi_{n_{j}}-\psi_{n_{k}}\right\rangle \\
\geq & \frac{\lambda-\lambda_{k-1}}{\lambda_{k-1}}\left\|\psi_{n_{k}}-\psi_{n_{j}}\right\|^{2}-\left\langle I^{\prime}\left(u_{n_{k}}\right)-I^{\prime}\left(u_{n_{j}}\right), \psi_{n_{j}}-\psi_{n_{k}}\right\rangle,
\end{aligned}
$$

so we have

$$
\frac{\lambda-\lambda_{k-1}}{\lambda_{k-1}}\left\|\psi_{n_{k}}-\psi_{n_{j}}\right\|^{2} \leq 2 M\left(\left\|J^{\prime}\left(u_{n_{k}}\right)\right\|+\| J^{\prime}\left(u_{n_{j}}\|+\| I^{\prime}\left(u_{n_{k}}\right)-I^{\prime}\left(u_{n_{j}}\right) \|\right),\right.
$$

which implies that

$$
\left\|\psi_{n_{k}}-\psi_{n_{j}}\right\| \rightarrow 0 \text { as } k, j \rightarrow \infty
$$

that is, $\left\{\psi_{n_{k}}\right\}$ is a Cauchy sequence in $H_{0}^{1}(\Omega, a)$.

In a similar way, we can prove that $\left\{\phi_{n_{k}}\right\}$ also has a Cauchy subsequence $\left\{\phi_{n_{k_{j}}}\right\}$. To sum up, we have showed that $\left\{u_{n_{k_{j}}}\right\}$ is a Cauchy sequence in $H_{0}^{1}(\Omega, a)$. Hence $J$ satisfies the $(P S)$ condition.

Step 2. We will prove that there exists $\delta_{0}>0$ such that for $\lambda \in\left(\lambda_{k}-\delta_{0}, \lambda_{k}\right)$, the first solution of problem (1) will be obtained by Theorem A.

For $u \in H_{k}^{\perp}$, from (8), (10) and (11) it follows that

$$
J(u) \geq \frac{1}{2}\left(1-\frac{\lambda}{\lambda_{k+1}}\right)\|u\|^{2}-C\left(1+\|u\|^{q}\right)-S\|h\|_{L^{2}}\|u\| .
$$

If $\lambda \in\left(\lambda_{k-1}, \lambda_{k}\right), 1-\frac{\lambda}{\lambda_{k+1}}>1-\frac{\lambda_{k}}{\lambda_{k+1}}>0$, which implies that there exists a constant $D_{1} \in \mathbb{R}$ such that $J(u) \geq D_{1}$ for all $u \in H_{k}^{\perp}$.

In addition, it follows from (2) and (A) that for any $M_{1}>0$, there exists $C_{1}>0$ such that

$$
F(x, t) \geq M_{1}|t|-C_{1}
$$

for $t \in \mathbb{R}$ and $x \in \Omega$. Set $\delta:=\lambda_{k}-\lambda>0$, for $u \in K S_{k}$, from (8), (9), (11), (14) and all the norms in a finite dimensional subspace are equivalent it follows that

$$
\begin{aligned}
J(u) & \leq \frac{1}{2}\left(1-\frac{\lambda}{\lambda_{k}}\right)\|u\|^{2}-\int_{\Omega} F(x, u) d x+S\|h\|_{L^{2}}\|u\| \\
& \leq \frac{\delta}{2 \lambda_{k}}\|u\|^{2}-M_{1} \int_{\Omega}|u| d x+C_{1}|\Omega|+S\|h\|_{L^{2}}\|u\| \\
& \leq \frac{\delta}{2 \lambda_{k}}\|u\|^{2}-M_{2}\|u\|+C_{1}|\Omega|
\end{aligned}
$$




$$
=\frac{\delta}{2 \lambda_{k}} K^{2}-M_{2} K+C_{1}|\Omega|,
$$

where $M_{2}$ is a positive constant. We fix $K=K_{1}>0$ such that $C_{1}|\Omega|-M_{2} K_{1}<$ $D_{1}-1$ and choose $0<\delta<2 \lambda_{k} / K_{1}^{2}=\delta_{0}$, then $J(u)<D_{1}$ for all $u \in K_{1} S_{k}$.

Let

$$
\Gamma_{1}=\left\{\gamma \in C^{0}\left(K_{1} B_{k} ; H_{0}^{1}(\Omega, a)\right):\left.\gamma\right|_{K_{1} S_{k}}=i d\right\} .
$$

Since $K_{1} S_{k}$ and $H_{k}^{\perp}$ link, by Theorem A we can obtain the first solution corresponding to a critical point at the critical level

$$
c_{1}=\inf _{\gamma \in \Gamma_{1}} \sup _{v \in K_{1} B_{k}} J(\gamma(v)) .
$$

Step 3. We shall obtain the second solution of problem (1) by Theorem A once more.

On one hand, for $\lambda \in\left(\lambda_{k}-\delta_{0}, \lambda_{k}\right)$ and $u \in H_{k-1}^{\perp}$, by (8), (10) and (11), we get

$$
J(u) \geq \frac{1}{2}\left(1-\frac{\lambda}{\lambda_{k}}\right)\|u\|^{2}-C\left(1+\|u\|^{q}\right)-S\|h\|_{L^{2}}\|u\|,
$$

which implies that there exists a constant $D_{2} \in \mathbb{R}$ such that $J(u) \geq D_{2}$ for all $u \in H_{k-1}^{\perp}$.

On the other hand, for $u \in H_{k-1}$, by estimates (8), (9) and (11), we have

$$
J(u) \leq \frac{1}{2}\left(1-\frac{\lambda}{\lambda_{k-1}}\right)\|u\|^{2}+C\left(1+\|u\|^{q}\right)+\|h\|_{L^{2}}\|u\| .
$$

For $\lambda \in\left(\lambda_{k-1}, \lambda_{k}\right)$, we have $1-\frac{\lambda}{\lambda_{k-1}}<0$, so for given $K_{1}$ in Step 2, we can find suitably large $\rho_{1}>K_{1}$ such that $J(u)<D_{2}$ for all $u \in \rho_{1} S_{k-1}$.

Let

$$
\Gamma_{2}=\left\{\gamma \in C^{0}\left(\rho_{1} B_{k-1} ; H_{0}^{1}(\Omega, a)\right):\left.\gamma\right|_{\rho_{1} S_{k-1}}=i d\right\} .
$$

Since $\rho_{1} S_{k-1}$ and $H_{k-1}^{\perp}$ link, by Theorem A we can obtained the second solution corresponding to a critical point at the level

$$
c_{2}=\inf _{\gamma \in \Gamma_{2}} \sup _{v \in \rho_{1} B_{k-1}} J(\gamma(v)) .
$$

Step 4. We show that $c_{2}<c_{1}$, which implies that these two solutions are different.

On one hand, from the estimates of Step 2, we have that $c_{1} \geq D_{1}$.

On the other hand, consider the continuous map $\gamma_{1}: \rho_{1} B_{k-1} \rightarrow H$ define by

$$
\gamma_{1}(u)= \begin{cases}u+\left(K_{1}^{2}-\|u\|^{2}\right)^{1 / 2} e_{k} & \|u\| \leq K_{1}, \\ u & K_{1} \leq\|u\| \leq \rho_{1},\end{cases}
$$

where $e_{k} \in E_{k}$ and $\left\|e_{k}\right\|=1$. Then we observe that the map $\gamma_{1} \in \Gamma_{2}$.

For $u \in H_{k-1}$, in a way similar to (15), one gets

$$
J(u) \leq \frac{1}{2}\left(1-\frac{\lambda}{\lambda_{k-1}}\right)\|u\|^{2}-\int_{\Omega} F(x, u) d x+S\|h\|_{L^{2}}\|u\|
$$




$$
\begin{aligned}
& \leq\left(\frac{\lambda_{k-1}-\lambda}{2 \lambda_{k-1}}\right)\|u\|^{2}-M_{1}\|u\|+C_{1}|\Omega|+S\|h\|_{L^{2}}\|u\| \\
& \leq\left(\frac{\lambda_{k-1}-\lambda}{2 \lambda_{k-1}}\right)\|u\|^{2}-M_{2}\|u\|+C_{1}|\Omega| .
\end{aligned}
$$

Since $\frac{\lambda_{k-1}-\lambda}{2 \lambda_{k-1}}<0$ and $C_{1}|\Omega|-M_{2} K_{1}<D_{1}$ by Step 2, we have $J(u)<D_{1}$ for all $u \in H_{k-1}$ with $\|u\| \geq K_{1}$. For $u \in K_{1} B_{k-1}$, let $\gamma_{1}(u)=u+\left(K_{1}^{2}-\|u\|^{2}\right)^{1 / 2} e_{k}$, then $\gamma_{1}(u) \in K_{1} S_{k}$, by Step 2, we have $J\left(\gamma_{1}(u)\right)<D_{1}$. Now we deduce that $\sup _{v \in \rho_{1} B_{k-1}} J\left(\gamma_{1}(v)\right)<D_{1}$, which implies that $c_{2}<D_{1} \leq c_{1}$. Our proof is completed.

Proof of Theorem 2. Observing the proof of Theorem 1, here we only need to prove that under our conditions there exists $\delta_{1}>0$ such that for $0<\delta<\delta_{1}$, we have $J(u)<D_{1}$ for all $u \in K_{1} S_{k}$. Other estimates are obtained by the same methods as in the proof of Theorem 1.

By (A) and (3), we can easily deduce that

$$
F(x, t) \geq-C_{2},
$$

for all $t \in \mathbb{R}$ and $x \in \Omega$ where $C_{2}>0$. Now we shall show that in the finite dimension space $H_{k}$, hypotheses (A) and (3) imply that

$$
\lim _{\|u\| \rightarrow \infty} \int_{\Omega} F(x, u) d x=\lim _{K \rightarrow \infty} \inf _{u \in K S_{k}} \int_{\Omega} F(x, u) d x=+\infty .
$$

First, we say that there exists a constant $\eta>0$ such that the set $\Omega_{u}=$ $\{x \in \Omega:|u(x)|>\eta\}$ has measure $\left|\Omega_{u}\right|>\eta$ for all $u \in S_{k}$. Actually, $H_{k}$ is a finite-dimensional subspace and the functions $u \in S_{k}$ are smooth, so they are uniformly bounded, that is, there exists $M_{3}>0$ such that $|u(x)| \leq M_{3}$ for all $x \in \Omega$. Suppose that for $\eta_{n} \rightarrow 0\left(\eta_{n}<1\right)$ there exists $\left\{u_{n}\right\} \subset S_{k}$ such that $\left|\Omega_{u_{n}}\right|<\eta_{n}$. On one hand, by (9), one has

$$
1 / \lambda_{k} \leq \int_{\Omega}\left|u_{n}\right|^{2} d x
$$

On the other hand,

$$
\begin{aligned}
\int_{\Omega}\left|u_{n}\right|^{2} d x & =\left(\int_{\Omega_{u_{n}}}\left|u_{n}\right|^{2} d x+\int_{\Omega \backslash \Omega_{u_{n}}}\left|u_{n}\right|^{2} d x\right) \\
& \leq\left(M_{3}^{2}\left|\Omega_{u_{n}}\right|+\eta_{n}^{2}\left|\Omega \backslash \Omega_{u_{n}}\right|\right) \\
& \leq \eta_{n}\left(M_{3}^{2}+|\Omega|\right) \\
& \rightarrow 0 .
\end{aligned}
$$

This is a contradiction.

Now, for any fixed $L>0$, setting $M_{4}=\left(L+|\Omega| C_{2}\right) \eta^{-1}$, by $(3)$, there exists $t_{0}>0$ such that $F(x, t)>M_{4}$ for $|t|>t_{0}$. For any $K>t_{0} / \eta$ and $u \in S_{k}$, one 
has $\Omega_{u} \subseteq\left\{x \in \Omega:|K u(x)|>t_{0}\right\}$. Thus we have

$$
\int_{|K u| \geq t_{0}} F(x, K u) d x \geq M_{4} \eta .
$$

From (19) it follows that

$$
\int_{|K u|<t_{0}} F(x, K u) d x \geq \int_{|K u|<t_{0}}-C_{2} d x \geq-C_{2}|\Omega| .
$$

To sum up, one has

$$
\int_{\Omega} F(x, K u) d x \geq M_{4} \eta-C_{2}|\Omega|=L,
$$

which implies that (20) holds by the arbitrariness of $L$.

Let $\lambda \in\left[\frac{\lambda_{k}+\lambda_{k-1}}{2}, \lambda_{k}\right)$ and $\mu:=\frac{\lambda_{k}-\lambda_{k-1}}{2 \lambda_{k-1}}>0$. Then

$$
1-\frac{\lambda}{\lambda_{k-1}}=\frac{\lambda_{k-1}-\lambda}{\lambda_{k-1}} \leq \frac{\lambda_{k-1}-\lambda_{k}}{2 \lambda_{k-1}}=-\mu .
$$

For $u=v+\phi \in H_{k-1} \oplus E_{k}$ with $\|u\|=K$, from (4) it follows that

$$
\begin{aligned}
J(u) & \leq \frac{1}{2}\left(1-\frac{\lambda}{\lambda_{k-1}}\right)\|v\|^{2}+\frac{1}{2}\left(1-\frac{\lambda}{\lambda_{k}}\right)\|\phi\|^{2}-\int_{\Omega} F(x, u) d x-\int_{\Omega} h u d x \\
& \leq \frac{\delta}{2 \lambda_{k}}\|\phi\|^{2}-\frac{\mu}{2}\|v\|^{2}-\int_{\Omega} F(x, u) d x+S\|h\|_{L^{2}}\|v\| \\
& \leq \frac{\delta}{2 \lambda_{k}}\|\phi\|^{2}-\frac{\mu}{2}\|v\|^{2}-\int_{\Omega} F(x, u) d x+\frac{\mu}{2}\|v\|^{2}+\frac{S^{2}}{2 \mu}\|h\|_{L^{2}}^{2} \\
(21) & \leq \frac{\delta}{2 \lambda_{k}}\|u\|^{2}-\int_{\Omega} F(x, u) d x+\frac{S^{2}}{2 \mu}\|h\|_{L^{2}}^{2} .
\end{aligned}
$$

By (20), we may fix $K_{1}>0$ such that $\frac{S^{2}}{2 \mu}\|h\|_{L^{2}}^{2}-\int_{\Omega} F(x, u) d x<D_{1}-1$ for all $\|u\| \geq K_{1}$. Then letting $0<\delta<\delta_{1}:=2 \lambda_{k} /\left(K_{1}\right)^{2}>0$, one gets $J(u)<D_{1}$ for $u \in K_{1} S_{k}$.

If $u \in H_{k-1}$, that is, $\phi=0$. By (21), there exists $K_{1}>0$ such that $\int_{\Omega} F(x, u) d x>\frac{S^{2}}{2 \mu}\|h\|_{L^{2}}^{2}-D_{1}+1$, so $J(u) \leq-\int_{\Omega} F(x, u) d x+\frac{S^{2}}{2 \mu}\|h\|_{L^{2}}^{2}<$ $D_{1}-1<D_{1}$ for all $u \in H_{k-1}$ with $\|u\|>K_{1}$.

The reminders are the same as in the proof of Theorem 1. Hence Theorem 2 holds.

Proof of Theorem 3. In the case $\lambda \in\left(\lambda_{k}, \lambda_{k+1}\right)$, from Step 1 in the proof of Theorem 1 it follows that the functional $J$ satisfies the $(P S)$ condition.

Step 1. The existence of the first solution of problem (1).

For $\lambda \in\left(\lambda_{k}, \lambda_{k+1}\right)$, by assumptions (A) and (5), we will prove the following estimates: there exist $\delta_{2}>0, G_{1}, K_{2}, E \in \mathbb{R}, \xi>0$ such that for $\lambda \in$ $\left(\lambda_{k}, \lambda_{k}+\delta_{2}\right)$, one has

$$
J(u)<G_{1} \text { for } u \in H_{k-1},
$$




$$
\begin{aligned}
& J(u)>G_{1} \text { for } u \in K_{2} S_{k-1}^{\perp}, \\
& J(u)>G_{1} \text { for } u \in H_{k}^{\perp},\|u\| \geq K_{2}, \\
& J(u)>E \text { for } u \in K_{2} B_{k-1}^{\perp}, \\
& J(u)<E \text { for } u \in \xi S_{k-1} .
\end{aligned}
$$

Thus let $X_{1}=H_{k-1}$ and $X_{2}=H_{k-1}^{\perp}$, by (22)-(26) we have the structure

$$
\sup _{\xi S_{k-1}} J(u)<E \leq \inf _{K_{2} B_{k-1}^{\perp}} J(u) \leq \sup _{\xi B_{k-1}} J(u)<G_{1} \leq \inf _{K_{2} S_{k-1}^{\perp}} J(u) .
$$

Then the first solution comes from Theorem B corresponding to critical point at the level $d_{1} \leq G_{1}$.

Now, we give the proofs of estimates above. For $u \in H_{k-1}$, by (8), (9) and (11), one has

$$
J(u) \leq \frac{1}{2}\left(1-\frac{\lambda}{\lambda_{k-1}}\right)\|u\|^{2}+C\left(1+\|u\|^{q}\right)+S\|h\|_{L^{2}}\|u\| .
$$

For $\lambda \in\left(\lambda_{k}, \lambda_{k+1}\right)$, we have $1-\frac{\lambda}{\lambda_{k-1}}<\frac{\lambda_{k-1}-\lambda_{k}}{\lambda_{k-1}}<0$. Then there exists $G_{1} \in \mathbb{R}$ such that (22) holds.

We claim that for $G_{1}$ above, there exist $K_{2}, \delta_{2}>0$ such that for every $\lambda \in\left(\lambda_{k}, \lambda_{k}+\delta_{2}\right)$, Eqs.(23) and (24) hold.

In addition, for $\lambda \in\left(\lambda_{k}, \lambda_{k+1}\right)$ and $u \in H_{k-1}^{\perp}$, by (8), (10) and (11), one gets

$$
\begin{aligned}
J(u) & \geq \frac{1}{2}\left(1-\frac{\lambda}{\lambda_{k}}\right)\|u\|^{2}-C\left(1+\|u\|^{q}\right)-S\|h\|_{L^{2}}\|u\| \\
& \geq \frac{1}{2}\left(1-\frac{\lambda_{k+1}}{\lambda_{k}}\right)\|u\|^{2}-C\left(1+\|u\|^{q}\right)-S\|h\|_{L^{2}}\|u\|,
\end{aligned}
$$

which implies that $J$ is bounded from below in any bounded subset of $H_{k-1}^{\perp}$, that is, for $K_{2}$, there exists $E \in \mathbb{R}$ satisfying (25). Further, by (27), for $E$ and $G_{1}$, there exists $\xi>0$ such that (26) holds.

It reminds to prove the claim above. Let $\lambda=\lambda_{k}+\delta$, by Eq.(16), (24) holds provided that $K_{2}$ is large enough (say $K_{2}>\tilde{K}$ ). Moreover, this value can be made independent of $\lambda$ once that $\delta$ is small enough.

Next, we will prove (23), that is, we will prove that there exists $K_{2}>0$ such that $J(u)>G_{1}$ for $u \in K_{2} S_{k-1}^{\perp}$.

Let $u=w+\phi \in H_{k-1}^{\perp}=H_{k}^{\perp} \oplus E_{k}$. Since $E_{k}$ is a finite dimension subspace, all the norms are equivalent, there exists $C_{0}>0$ such that $\|\phi\| \leq C_{0}\|\phi\|_{L^{1}}$ for all $\phi \in E_{k}$. By (A) and (5), we have for $\left(S\|h\|_{L^{2}}+1\right) C_{0}>0$, there exists constant $C_{4}$ such that

$$
-F(x, t) \geq\left(\left(S\|h\|_{L^{2}}+1\right) C_{0}\right)|t|-C_{4}
$$


for all $t \in \mathbb{R}$ and $x \in \Omega$. By (10) and (28), one has

$$
\begin{aligned}
J(u)= & \frac{1}{2} \int_{\Omega}\left(|\nabla(w+\phi)|^{2}-\lambda(w+\phi)^{2}\right) d x-\int_{\Omega} F(x, w+\phi) d x-\int_{\Omega} h(w+\phi) d x \\
\geq & \frac{\lambda_{k+1}-\left(\lambda_{k}+\delta\right)}{2 \lambda_{k+1}}\|w\|^{2}-\frac{\delta}{2 \lambda_{k}}\|\phi\|^{2} \\
& +\left(S\|h\|_{L^{2}}+1\right) C_{0}\|\phi\|_{L^{1}}-\left(S\|h\|_{L^{2}}+1\right) C_{0}\|-w\|_{L^{1}}-\|h\|_{L^{2}}\|\phi\|_{L^{2}} \\
& -\|h\|_{L^{2}}\|w\|_{L^{2}}-C_{4}|\Omega| \\
\geq & \frac{\lambda_{k+1}-\left(\lambda_{k}+\delta\right)}{2 \lambda_{k+1}}\|w\|^{2}-\frac{\delta}{2 \lambda_{k}}\|\phi\|^{2} \\
& +\left(S\|h\|_{L^{2}}+1\right)\|\phi\|-|\Omega|^{1 / 2}\left(S\|h\|_{L^{2}}+1\right) C_{0}\|w\|_{L^{2}}-S\|h\|_{L^{2}}\|\phi\| \\
& -S\|h\|_{L^{2}}\|w\|-C_{4}|\Omega| \\
\geq & \frac{\lambda_{k+1}-\left(\lambda_{k}+\delta\right)}{2 \lambda_{k+1}}\|w\|^{2}-\frac{\delta}{2 \lambda_{k}}\|\phi\|^{2}+\|\phi\|-C_{5}\|w\|-C_{6},
\end{aligned}
$$

where $C_{5}=|\Omega|^{1 / 2} S\left(S\|h\|_{L^{2}}+1\right) C_{0}+S\|h\|_{L^{2}}, C_{6}=C_{4}|\Omega|$. Since

$$
\begin{aligned}
\left(1-\frac{\delta}{2 \lambda_{k}}\|u\|\right)\|u\| & \leq\|w\|+\|\phi\|-\frac{\delta}{2 \lambda_{k}}\left(\|\phi\|^{2}+\|w\|^{2}\right) \\
& \leq\left(1-\frac{\delta}{2 \lambda_{k}}\|\phi\|\right)\|\phi\|+\|w\| .
\end{aligned}
$$

Let $\delta \leq\left(\lambda_{k+1}-\lambda_{k}\right) / 2$, Eq.(29) becomes

$$
\begin{aligned}
& J(w+\phi) \geq \frac{\lambda_{k+1}-\left(\lambda_{k}+\delta\right)}{2 \lambda_{k+1}}\|w\|^{2}-C_{5}\|w\|-C_{6}-\|w\|+\left(1-\frac{\delta}{2 \lambda_{k}}\|u\|\right)\|u\| \\
& \text { (30) } \quad \geq \frac{\lambda_{k+1}-\lambda_{k}}{4 \lambda_{k+1}}\|w\|^{2}-\left(C_{5}+1\right)\|w\|-C_{6}+\left(1-\frac{\delta}{2 \lambda_{k}}\|u\|\right)\|u\| .
\end{aligned}
$$

Since $\frac{\lambda_{k+1}-\lambda_{k}}{4 \lambda_{k+1}}>0$, there exists $C_{7} \in \mathbb{R}$ such that $\frac{\lambda_{k+1}-\lambda_{k}}{4 \lambda_{k+1}}\|w\|^{2}-C_{5}\|w\|-C_{6} \geq$ $C_{7}$. From this and (30) it follows that

$$
J(u) \geq\left(1-\frac{\delta}{2 \lambda_{k}}\|u\|\right)\|u\|+C_{7} .
$$

Now we can choose $K_{2}$ large enough such that $K_{2}+C_{7}>G_{1}+1$ and (24) holds, then for $0<\delta<\min \left\{2 \lambda_{k} / K_{2}^{2},\left(\lambda_{k+1}-\lambda_{k}\right) / 2\right\}=\delta_{2}$ and $u \in K_{2} S_{k-1}^{\perp}$, one gets $J(u)>G_{1}$, that is, (23) holds.

Step 2. The existence of the second solution of problem (1).

For $\lambda \in\left(\lambda_{k}, \lambda_{k}+\delta_{2}\right)$ and $u \in H_{k}^{\perp}$, by (8), (10) and (11), we get

$$
J(u) \geq \frac{1}{2}\left(1-\frac{\lambda}{\lambda_{k+1}}\right)\|u\|^{2}-C\left(1+\|u\|^{q}\right)-S\|h\|_{L^{2}}\|u\|,
$$

which implies that there exists a constant $G_{2} \in \mathbb{R}$ such that $J(u) \geq G_{2}$ for all $u \in H_{k}^{\perp}$. 
For $u \in H_{k}$, by (8), (9) and (11), we get

$$
J(u) \leq \frac{1}{2}\left(1-\frac{\lambda}{\lambda_{k}}\right)\|u\|^{2}+C\left(1+\|u\|^{q}\right)+S\|h\|_{L^{2}}\|u\| .
$$

then for $K_{2}$ in Step 1, we can find $\rho_{2}>K_{2}$ such that $J(u)<G_{2}$ for all $u \in \rho_{2} S_{k}$.

Let

$$
\Gamma_{3}=\left\{\gamma \in C^{0}\left(\rho_{2} B_{k} ; H_{0}^{1}(\Omega)\right):\left.\gamma\right|_{\rho_{2} S_{k}}=i d\right\} .
$$

Since $\rho_{2} S_{k}$ and $H_{k}^{\perp}$ link, then for every $\lambda \in\left(\lambda_{k}, \lambda_{k}+\delta_{2}\right)$, the second solution is obtained by Theorem A corresponding to critical point at the level

$$
d_{2}=\inf _{\gamma \in \Gamma_{3}} \sup _{v \in \rho_{2} B_{k}} J(\gamma(v)) .
$$

In order to distinguish two solutions obtained above, we need the following lemma.

Lemma A ([7]). For $\rho>K>0$, the set $\rho S_{k}$ links the set

$$
\hat{W}=\left\{u \in H_{k}^{\perp}:\|u\| \geq K\right\} \cup K S_{k-1}^{\perp} .
$$

Step 3. we will prove that these two solutions are distinct.

For any map $\gamma \in \Gamma_{3}$, from the proof of Step 1, we can choose $\rho_{2}>K_{2}$, one has that the image of $\gamma$ either intersects $K_{2} S_{k-1}^{\perp}$ or has a point $u \in H_{k}^{\perp}$ with $\|u\| \geq K_{2}$ by Lemma A. This implies that $\sup _{v \in \rho_{2} B_{k}} J(\gamma(v))>G_{1}$ by estimates (23) and (24). Then $d_{2}>G_{1} \geq d_{1}$, which shows that these two solutions are different. Our proof is completed.

Proof of Theorem 4. Under assumptions (A), (6) and (7), we only need to prove that there exist $K_{2}, \delta_{3}>0$ such that for $\lambda \in\left(\lambda_{k}, \lambda_{k}+\delta_{3}\right)$ and $G_{1}$ given in the proof of Theorem 3, one has

$$
J(u)>G_{1}
$$

for $u \in K_{2} S_{k-1}^{\perp}$.

First we give a conclusion which is similar to Lemma 3 in [17]. Under the assumptions on $F$, there exist a constant $C_{8}$ and $G \in C(\mathbb{R}, \mathbb{R})$ which is subadditive, that is,

$$
G(s+t) \leq G(s)+G(t)
$$

for all $s, t \in \mathbb{R}$, and coercive, that is,

$$
G(s) \rightarrow+\infty
$$

as $|s| \rightarrow \infty$, and satisfies that

$$
G(s) \leq|s|+4
$$

for all $s \in \mathbb{R}$, such that

$$
-F(x, s) \geq G(s)-C_{8}
$$

for all $s \in \mathbb{R}$ and $x \in \bar{\Omega}$. 
In fact, since $-F(x, s) \rightarrow+\infty$ as $|s| \rightarrow \infty$ uniformly for $x \in \bar{\Omega}$, there exists a sequence of positive integers $\left(n_{k}\right)$ with $n_{k+1}>2 n_{k}$ for all positive integers $k$ such that

$$
-F(x, s) \geq k
$$

for all $|s| \geq n_{k}$ and all $x \in \bar{\Omega}$. Let $n_{0}=0$ and define

$$
G(s)=k+2+\frac{|s|-n_{k-1}}{n_{k}-n_{k-1}}
$$

for $n_{k-1} \leq|s|<n_{k}$, where $k \in \mathbb{N}$. By the definition of $G$, we have

$$
k+2 \leq G(s) \leq k+3
$$

for all $n_{k-1} \leq|s|<n_{k}$. By $(6)$ and $F \in C^{1}(\bar{\Omega} \times \mathbb{R}, \mathbb{R})$, there exists $C_{F}>0$ such that

$$
-F(x, s) \geq-C_{F}
$$

for all $(x, s) \in \Omega \times \mathbb{R}$, which implies that

$$
-F(x, s) \geq G(s)-C_{8},
$$

where $C_{8}=C_{F}+4$. Indeed, when $n_{k-1} \leq|s|<n_{k}$ for some $k \geq 2$, one has, by (37) and (39),

$$
-F(x, s) \geq k-1 \geq G(s)-4 \geq G(s)-C_{8}
$$

for all $x \in \bar{\Omega}$. When $|s|<n_{1}$, we have, by (40) and (39),

$$
-F(x, s) \geq-C_{F}=4-C_{8} \geq G(s)-C_{8}
$$

for all $x \in \bar{\Omega}$.

It is obvious that $G$ is continuous and coercive. Moreover one has

$$
G(s) \leq|s|+4
$$

for all $s \in \mathbb{R}$. In fact, for every $s \in \mathbb{R}$ there exists $k \in \mathbb{N}$ such that

$$
n_{k-1} \leq|s|<n_{k},
$$

which implies that

$$
G(s) \leq(k-1)+4 \leq n_{k-1}+4 \leq|s|+4
$$

for all $s \in \mathbb{R}$ by (39) and the fact that $n_{k} \geq k$ for all integers $k \geq 0$.

Now we only need to prove the subadditivity of $G$. Let

$$
n_{k-1} \leq|s|<n_{k}, \quad n_{j-1} \leq|t|<n_{j}
$$

and $m=\max \{k, j\}$. Then we have

$$
|s+t| \leq|s|+|t|<n_{k}+n_{j} \leq 2 n_{m}<n_{m+1} .
$$

Hence we obtain, by (39),

$$
G(s+t) \leq m+4 \leq k+2+j+2 \leq G(s)+G(t),
$$

which shows that $G$ is subadditive. 
For $u:=w+\phi \in H_{k-1}^{\perp}=H_{k}^{\perp} \oplus E_{k}$, let $0<\delta<\left(\lambda_{k+1}-\lambda_{k}\right) / 2$, by $(10),(6)$, (36), (33) and (35), one gets

$$
\begin{aligned}
J(w+\phi)= & \frac{1}{2} \int_{\Omega} a(x)|\nabla(w+\phi)|^{2} d x-\frac{\lambda}{2} \int_{\Omega}(w+\phi, w+\phi) d x \\
& -\int_{\Omega} F(x, w+\phi) d x-\int_{\Omega}(h, w+\phi) d x \\
\geq & \frac{\lambda_{k+1}-\left(\lambda_{k}+\delta\right)}{2 \lambda_{k+1}}\|w\|^{2}-\frac{\delta}{2 \lambda_{k}}\|\phi\|^{2} \\
& +\int_{\Omega} G(\phi+w) d x-C_{8}|\Omega|-\int_{\Omega}(h, w) d x \\
\geq & \frac{\lambda_{k+1}-\left(\lambda_{k}+\delta\right)}{2 \lambda_{k+1}}\|w\|^{2}-\frac{\delta}{2 \lambda_{k}}\|\phi\|^{2} \\
& +\int_{\Omega} G(\phi) d x-\int_{\Omega} G(-w) d x-C_{8}|\Omega|-\int_{\Omega}(h, w) d x \\
\geq & \frac{\lambda_{k+1}-\lambda_{k}}{4 \lambda_{k+1}}\|w\|^{2}-\frac{\delta}{2 \lambda_{k}}\|\phi\|^{2} \\
& +\int_{\Omega} G(\phi) d x-\int_{\Omega}(|w|+4) d x-C_{8}|\Omega|-\int_{\Omega}(h, w) d x \\
\geq & \frac{\lambda_{k+1}-\lambda_{k}}{4 \lambda_{k+1}}\|w\|^{2}-\frac{\delta}{2 \lambda_{k}}\|u\|^{2} \\
& +\int_{\Omega} G(\phi) d x-\left(S|\Omega|+S\|h\|_{L^{2}}\right)\|w\|-C_{9} \\
= & g(w)+\int_{\Omega} G(\phi) d x-\frac{\delta}{2 \lambda_{k}}\|u\|^{2},
\end{aligned}
$$

where $g(w)=\frac{\lambda_{k+1}-\lambda_{k}}{4 \lambda_{k+1}}\|w\|^{2}-\left(S|\Omega|+S\|h\|_{L^{2}}\right)\|w\|-C_{9}, C_{9}=\left(4+C_{8}\right)|\Omega|$. Since $\phi \in E_{k}, H_{k}$ is a finite-dimensional subspace, and $G$ is coercive, from the proof of (20), one can get

$$
\lim _{\|\phi\| \rightarrow \infty} \int_{\Omega} G(\phi) d x=+\infty
$$

that is, $\int_{\Omega} G(\phi) d x$ is coercive on $H_{k}$. Since $\frac{\lambda_{k+1}-\lambda_{k}}{4 \lambda_{k+1}}>0$, so $g$ is coercive on $H_{k}^{\perp}$. Moreover, $\int_{\Omega} G(\phi) d x$ and $g(w)$ are bounded from below respectively in $E_{k}$ and $H_{k}^{\perp}$, then $g(w)+\int_{\Omega} G(\phi) d x$ is coercive on $H_{k-1}^{\perp}=H_{k}^{\perp} \oplus E_{k}$. Now we can choose $K_{2}$ large enough such that $g(w)+\int_{\Omega} G(\phi) d x>G_{1}+1$ for all $u \in H_{k-1}^{\perp}$ with $\|u\| \geq K_{2}$, and (24) holds. Then for $0<\delta<\min \left\{2 \lambda_{k} / K_{2}^{2},\left(\lambda_{k+1}-\lambda_{k}\right) / 2\right\}=\delta_{3}$, we have $J(u)>G_{1}$ for $u \in K_{2} S_{k-1}^{\perp}$.

The reminders are the same as in the proof of Theorem 3. Hence Theorem 4 holds. 


\section{References}

[1] M. Badiale and D. Lupo, Some remarks on a multiplicity result by Mawhin and Schmitt, Acad. Roy. Belg. Bull. Cl. Sci. (5) 65 (1989), no. 6-9, 210224.

[2] P. Caldiroli and R. Musina, On a variational degenerate elliptic problem, Nonlinear Differential Equations Appl. 7 (2000), no. 2, 187-99.

[3] R. Chiappinelli and D. G. de Figueiredo, Bifurcation from infinity and multiple solutions for an elliptic system, Differential Integral Equations 6 (1993), no. 4, 757-771.

[4] R. Chiappinelli, J. Mawhin, and R. Nugari, Bifurcation from infinity and multiple solutions for some Dirichlet problems with unbounded nonlinearities, Nonlinear Anal. 18 (1992), no. 12, 1099-1112.

[5] R. Dautary and J. L. Lions, Mathematical Analysis and Numerical Methods for Science and Technology. Vol. 1., Springer-Verlag, Berlin, 1990.

[6] P. De Nápoli and M. Mariani, Three solutions for quasilinear equations in $\mathbb{R}^{n}$ near resonance, Proceedings of the USA-Chile Workshop on Nonlinear Analysis (Viña del Mar-Valparaiso, 2000), 131-140, Electron. J. Differ. Equ. Conf., 6, Southwest Texas State Univ., San Marcos, TX, 2001.

[7] Francisco Odair de Paiva and Eugenio Massa, Semilinear elliptic problems near resonance with a nonprincipal eigenvalue, J. Math. Anal. Appl. 342 (2008), no. 1, 638-650.

[8] M. Frigon, On a new notion of linking and application to elliptic problems at resonance, J. Differential Equations 153 (1999), no. 1, 96-120.

[9] D. Lupo and M. Ramos, Some multiplicity results for two-point boundary value problems near resonance, Rend. Sem. Mat. Univ. Politec. Torino 48 (1990), no. 2, 125-135 (1992).

[10] T. F. Ma and M. L. Pelicer, Perturbations near resonance for the p-Laplacian in $\mathbb{R}^{N}$, Abstr. Appl. Anal. 7 (2002), no. 6, 323-334.

[11] T. F. Ma, M. Ramos, and L. Sanchez, Multiple solutions for a class of nonlinear boundary value problems near resonance: a variational approach, Proceedings of the Second World Congress of Nonlinear Analysts, Part 6 (Athens, 1996). Nonlinear Anal. 30 (1997), no. 6, 3301-3311.

[12] A. Marino, A. M. Micheletti, and A. Pistoia, A nonsymmetric asymptotically linear elliptic problem, Topol. Methods Nonlinear Anal. 4 (1994), no. 2, 289-339.

[13] J. Mawhin and K. Schmitt, Landesman-Lazer type problems at an eigenvalue of odd multiplicity, Results Math. 14 (1988), no. 1-2, 138-146.

[14] Nonlinear eigenvalue problems with the parameter near resonance, Ann. Polon. Math. 51 (1990), 241-248.

[15] P. H. Rabinowitz, Minimax methods in critical point theory with applications to differential equations, CBMS Reg. Conf. Ser. Math. vol. 65, Conference Board of the Mathematical Sciences, Washington, DC, 1986.

[16] M. Ramos and L. Sanchez, A variational approach to multiplicity in elliptic problems near resonance, Proc. Roy. Soc. Edinburgh Sect. A 127 (1997), no. 2, 385-394.

[17] C. L. Tang and X. P. Wu, Periodic solutions for second order systems with not uniformly coercive potential, J. Math. Anal. Appl. 259 (2001), no. 2, 386-397.

HONG-Min SuO

School of Mathematics and Statistics

SOUTHWEST UNIVERSITY

Chongqing 400715, P. R. China

AND

College of Science

GuiZhou University For NAtionalities

Guiyang, 550025, P. R. China 
Chun-Lei Tang

School of Mathematics and Statistics

SOUTHWEST UNIVERSITY

Chongqing 400715, P. R. China

E-mail address: tangcl@swu.edu.cn 